\title{
Attenuating the haptic horizontal-vertical curvature illusion
}

\author{
Morton A. Heller, Anne D. McClure Walk, Rita Schnarr, \\ Stephanie Kibble, Brett Litwiller, and Cassie Ambuehl \\ Eastern Illinois University, Charleston, Illinois
}

\begin{abstract}
In a number of experiments, blindfolded subjects traced convex curves whose verticals were equal to their horizontal extent at the base. Overestimation of verticals, as compared with horizontals, was found, indicating the presence of a horizontal-vertical illusion with haptic curves, as well as with visible curves. Experiment 1 showed that the illusion occurred with stimuli in the frontal plane and with stimuli that were flat on the table surface in vision and touch. In the second experiment, the stimuli were rotated, and differences between vision and touch were revealed, with a stronger illusion in touch. The haptic horizontal-vertical illusion was virtually eliminated when the stimuli were bimanually touched using free exploration at the body midline, but a strong illusion was obtained when curves were felt with two index fingers or with a single hand at the midline. Bimanual exploration eliminated the illusion for smaller 2.5 - through $10.2-\mathrm{cm}$ stimuli, but a weakened illusion remained for the largest $12.7-\mathrm{cm}$ patterns. The illusion was present when the stimuli were bimanually explored in the left and right hemispace. Thus, the benefits of bimanual exploration derived from the use of the two hands at the body midline combined with free exploration, rather than from bimanual free exploration per se. The results indicate the importance of haptic exploration at the body midline, where the body can serve as a familiar reference metric for size judgments. Alternative interpretations of the results are discussed, including the impact of movement-based heuristics as a causal factor for the illusion. It was suggested that tracing the curve's peak served to bisect the curve in haptics, because of the change in direction.
\end{abstract}

Prior research revealed a robust overestimation of the vertical dimension of haptic curves, as compared with the horizontal, when the vertical was equal to the horizontalthat is, the width at the base (Heller et al., 2008). Conceivably, the overestimation in haptics could have derived from radial scanning, since the tangible stimuli were flat on the tabletop. This meant that tracing the sides of the curves involved arm motions that were nearly radial and were directed toward the body. Radial scanning has been used to explain overestimation of vertical lines for inverted-T and L shapes (see Heller, Brackett, Salik, Scroggs, \& Green, 2003; Hollins \& Goble, 1988; Jones \& Lederman, 2006; Wong, 1977).

Furthermore, there is clear evidence that the haptic horizontal-vertical illusion is partially configurational; that is, it is influenced by the nature of the pattern, since it is much stronger for inverted-T than for L shapes (see Millar, 2006). The inverted-T shape combines two illusory phenomena. First, it involves the horizontal-vertical illusion and radial/tangential scanning effects. Second, it encompasses the bisection illusion, since the vertical line alters the bottom of the shape. The bisection of the bottom of the inverted-T shape could explain the generally reported underestimation of the horizontal. The overestimation of the continuous line in inverted-T shapes is not found if the pattern is rotated $90^{\circ}$ (Tedford \& Tudor, 1969).
The present experiments were designed to study the roles of radial scanning and other factors involving haptic exploration in the overestimation of the verticals of curves and the relative underestimation of the horizontals of tangible curves. In addition, the aim was to clarify the factors responsible for the illusion and determine whether it is possible to attenuate or eliminate it.

Illusions are important from a number of theoretical perspectives, and it is important to discover whether they can be eliminated and how this can be accomplished. From the ecological perspective, illusions take place because of factors such as impoverished presentation conditions, abnormal or artificial stimuli, or other abnormal circumstances. On the ecological view, normal perception is presumably veridical. Others view illusions as typical of our perceptual experience. Furthermore, illusions may occur in vision and in touch, but perhaps for different reasons. Many visual illusions may be overcome with feedback, but this is often a difficult and slow process. In addition, there is evidence that there are large individual differences in susceptibility to illusory misperception. If it were possible to eliminate the horizontal-vertical illusion, this would be consistent with the ecological theoretical framework. It is important that Millar and Al-Attar (2000) found that exploration with the right index finger at the midline served to eliminate the horizontal-vertical illusion with an L shape. This occurred

M. A. Heller, maheller@eiu.edu 
when the vertical line from the $\mathrm{L}$ was aligned with the body midline. Thus, the manner in which stimuli are explored determined judgments of extent.

The haptic Müller-Lyer illusion may be diminished with the use of two hands for exploration (see Heller et al., 2005). Perhaps bimanual exploration would have similar effects on the horizontal-vertical illusion. Bimanual free exploration might allow subjects the use of optimal exploration methods. Exploration at the midline might allow subjects to feel their bodies with their elbows as they explore patterns with both hands, and the body is a familiar size. They could then compare the explored illusory configuration with this known body metric to estimate stimulus size. This placement could also provide more stable haptic exploration, since the body can be used as a support for the arms during haptic examination of stimuli.

Moreover, the horizontal-vertical illusion is greatly weakened when subjects are prevented from moving their whole arms (Heller, Calcaterra, Burson, \& Green, 1997). Arm motion may not be an optimal exploration method for accurate perception of small stimuli, where exploration of the hand and fingers may be more suitable. The results of a number of studies suggest that illusory misperception may often be related to exploration that involves an arm space.

Illusory misperception may also be a consequence of limiting subjects to the use of a single digit of one hand for haptic exploration. Use of a single finger for tracing yields radial motion when subjects feel inverted-T or $\mathrm{L}$ patterns where the vertical extent converges upon the body. These radial motions of the finger and arm are judged to be longer than tangential movements that do not converge upon the body (Wong, 1977). Wong provided evidence that radial movements are executed more slowly and are judged as longer. Poor or limited conditions for the observation of stimuli can yield misperception in vision and, presumably, in touch as well (see Gibson, 1979).

The presence or absence of a horizontal-vertical illusion is of substantial theoretical importance, especially given the argument that it is merely an artifact of radial scanning (Day \& Avery, 1970). According to Day and Avery, the illusion is not a basic function of haptic space, since the illusion was not found when stimuli were gravitationally vertical and exploration was not radial. Day and Avery argued that the haptic illusion is limited to conditions involving radial scanning. The haptic horizontal-vertical illusion involving inverted-T and L shapes was altered substantially by orienting stimuli in the frontal plane, with the elimination of radial scanning (Heller et al., 2003). Using the frontal position, as on a wall or an LCD display, subjects showed a small negative haptic illusion and overestimated horizontals, as compared with verticals. These results were consistent with those reported by Day and Avery, who asserted that the horizontal-vertical illusion is absent in haptic space and is an artifact of radial scanning (see also Day \& Wong, 1971; Heller et al., 1997). All scanning is tangential when stimuli are oriented in the frontal plane. Day and Avery proposed that the horizontal-vertical illusion occurs for different reasons in the two modalities of vision and touch.

The ecological perspective assumes intersensory equivalence, and so the present investigation takes on some im- portance. Should differences appear between vision and touch, we would have empirical evidence that raises problems for a simple version of the ecological view. Note that Gentaz and Hatwell (2004) also concluded that the haptic illusion is linked to exploration and the nature of movements, whereas the visual illusion functions differently and is related to the anisotropy of the visual field. Both modalities are affected by bisection. Thus, should one be able to further demonstrate that the illusion is not present in touch (but is present in vision), this also would pose a problem for some ecological perspectives. The difficulty would exist if conditions for visual examination were optimal, yet the illusion persisted in vision, but not in touch. If it could be shown that the presence or absence of the illusion depends on skilled exploration, or optimal exploration methods, one might have a rejoinder to the position of Day and Avery (1970).

Gibson $(1966,1979)$ proposed that illusions derive from impoverished stimulation, artificial stimuli, inadequate viewing conditions, or sensory restriction (as to a single modality). From this perspective, illusions represent perceptual errors, and people have evolved to perceive the world accurately. Presumably, perceptual accuracy is more likely to yield survival and has evolutionary value. However, Jackson and Cormack (2008) have recently claimed that there can be survival value in systematic illusions. On their view, sensitivity to the vertical in drawings could be derived in some manner from sensitivity to the gravitational vertical in large-scale space. In mobility in space, overestimation of the vertical represents a falling cost avoidance mechanism, owing to falling risk. However, the presence of any illusory misperception is problematic for the ecological position if the illusion occurs under optimal examination and presentation conditions.

One ecological assertion has been that illusions derive from artificial stimuli, such as line drawings. In touch, the equivalent would be raised-line drawings, and one could make the argument that these are not natural stimuli. However, there are reports that the horizontalvertical illusion occurs with sight of naturally occurring curved objects, such as the St. Louis Arch (Coren \& Girgus, 1978; Heelan, 1983). The St. Louis Arch is the same height and width at the base, yet it looks much taller than it is wide. Heller et al. (2008) reported similar perceptual overestimation of verticals in haptic size estimates of raised line curves and $\mathrm{L}$ patterns, and the illusion was found with solid wooden inverted-T and L shapes (Heller et al., 2003).

The experiments in this report were designed to determine whether the horizontal-vertical illusion occurs in a similar manner in vision and touch and to ascertain whether it is possible to eliminate it. In Experiment 1, subjects traced swell paper convex tangible curves with the index fingers of their preferred hands. Other groups used vision. All of the curves in the present study had vertical extents equal to their horizontals. The curves either were flat on the table surface or were in the frontal orientation. The stimuli in the frontal position were gravitationally vertical, and this eliminated any possibility of radial scanning. As one traces a convex curve that is flat on a table 
surface, scanning of the sides of the curve involves arm motions directed toward one's body, and the consequent arm motion is forward and backward. If the illusion were explainable solely in terms of radial-tangential scanning, with overestimation of scans where motions converge on the body, one would expect that the illusion would disappear when the curves were in the frontal position. In Experiment 2 , subjects viewed or felt curves with baselines that were upright or tilted $+90^{\circ}$. This was a further test of the possible role of scanning toward the body, since the baseline was vertical in the rotated stimuli. Experiment 3 examined the possible role of a baseline in the horizontalvertical curvature illusion.

Experiment 4 allowed subjects the use of both hands to feel the stimuli, with no constraints on manner of touch at the midline. A second group of subjects felt the stimuli with the index fingers of both hands at the midline. This experiment was designed to evaluate the importance of haptic coding with respect to the body midline combined with free exploration. At this location, the body could represent a familiar spatial metric and frame of reference for judgments of extent. Ballesteros and her colleagues have shown that haptic symmetry is more readily detected when stimuli are bimanually explored at the midline (Ballesteros, Manga, \& Reales, 1997; see also Millar, 2006). Note that Heller et al. (2005) found that bimanual exploration at the midline nearly eliminated the Müller-Lyer illusion. Experiment 5 was designed to test the idea that free exploration could explain the possible elimination of the illusion, and subjects felt the stimuli with either their left hands or their right hands at the midline. If bimanual exploration were critical for eliminating the illusion, overestimation of verticals, as compared with horizontals, should be found in Experiment 5. Experiment 6, the final experiment, allowed bimanual free exploration, but in the left or right hemispace. This experiment tested the importance of combining the effects of bimanual exploration with midline placement.

\section{EXPERIMENT 1 \\ The Effect of Position on the Haptic Horizontal-Vertical Curvature Illusion}

This experiment compared vision and touch and examined the impact of position on the illusion. Two groups of subjects were haptically exposed to convex tangible patterns that were either gravitationally vertical in the frontal plane or flat on the table surface. If the haptic illusion were solely explicable in terms of overestimation of radially scanned lines, the illusion should disappear for the group with stimuli in the frontal position. Two additional groups of subjects used vision, but with the stimuli either gravitationally vertical or flat on the table surface, as in the haptic groups.

\section{Method}

Subjects. There were two groups of blindfolded subjects, with 12 in each group (total $N=24 ; 15$ females, 9 males). Twelve of these subjects had stimuli in the frontal plane (6 females and 6 males), and the other 12 had the stimuli flat on the table ( 9 females and 3 males). Two more groups of subjects (flat [10 females and 2 males] vs. frontal [ 8 females and 4 males]) performed the task, but with vision of the stimuli. All the subjects were experimentally naive and were recruited on an undergraduate campus. The subjects were all strongly right-handed, as determined by answering "right hand" when asked which hand was used for the following activities: brush your teeth, throw a ball, write, draw, eat with a soup spoon, and cut with scissors.

Stimuli and Apparatus. The stimuli were raised tangible convex curves on swell paper, with their verticals equal to their horizontals at $2.54,5.08,7.62,10.18$, and $12.7 \mathrm{~cm}(M$ overall size $=7.6 \mathrm{~cm}$; see Figure 1). If one photocopies a pattern on swell paper, anything black on the surface will rise up if the paper is heated. The tangible and visible stimuli were the precise configurations that can be seen in Figure 1.

Size judgments were obtained using a pair of wooden tangible rulers. Markings on the rulers were visible to the experimenters, but not to the subjects in the haptic groups. Moreover, the markings were not tangible. The rulers were mounted to a shelf, and a clipboard was fastened to the front for the group of subjects with the stimuli in the frontal plane. Ruler spatial orientation was always like that of the stimulus dimension being judged. Both groups used the same horizontal ruler for judgments of the horizontal (see Figure 2). The group with the stimuli that were flat on the table surface beneath the shelf had a ruler that was oriented radially, so that they pulled the ruler out toward themselves to make vertical judgments. The stimuli were placed beneath the shelf for these subjects (see Figure 2). The apparatus was altered for the subjects when the stimuli were in the frontal position. For these subjects, a gravitationally vertical ruler extended from the top surface of the shelf for judgments of the verticals (Figure 2, bottom). Plain, unmarked wooden slats were used for the visual group.

Design and Procedure. The experiment was a between-within mixed design, with independent groups for position (flat or frontal) and modality (haptics vs. vision), with repeated measures on size (5), orientation (horizontal or vertical), and trials (4). The subjects wore blindfolds throughout in all the haptic groups reported in this article. Each trial block included vertical and horizontal judgments of all sizes of stimuli. Half of the subjects made vertical judgments before judging the horizontal dimension of each stimulus; the others used the reverse sequence. The subjects used their right index fingers to trace the tangible curves for as long as they wished and used their left hands to adjust the appropriate haptic ruler. Time limits were not imposed, and the subjects were allowed to feel the lines and the ruler at the same time, if they wished. The subjects could trace the lines as many times as desired but were restricted to the right index finger for tracing. After making a size judgment of the vertical or horizontal, the subjects were told to feel the entire stimulus again before judgment of the other dimension. This method was adopted in order to ensure that the subjects responded to the entire pattern when making their judgments of the vertical or horizontal. Feedback on performance was not given in all the experiments reported here.

The subjects were told that they were to feel (or view) tangible line patterns and judge their size, using tangible rulers. In the touch groups, they were instructed that they could not move their index fingers from the lines that they felt and that they could not trace between the endpoints of the stimuli. They were told that they should judge the peak height of the patterns from their tops to the base and the horizontals across their widest point at their base. The nature of the task was visually illustrated with an irregularly shaped flexible ruler (shown in Figure 1B). The ruler was bent to an approximation of the illustration in order to aid instruction of the subjects. They were told that the flexible ruler was not the stimulus that they would feel in the experiment but was there to be sure that they understood the nature of the judgments that they would make. We wanted to ensure that the subjects would not attempt to judge the lengths of the lines themselves.

The subjects were instructed about the appropriate haptic ruler to use for each judgment. Thus, when judging verticals, they were 

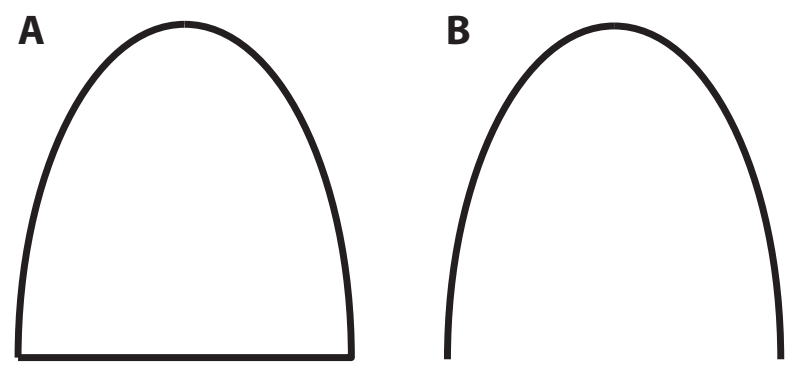

C

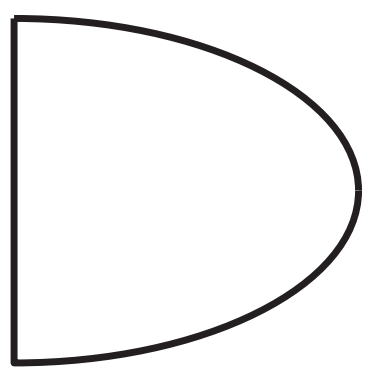

D

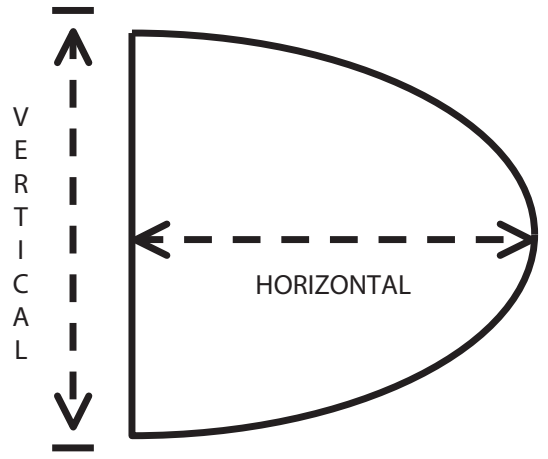

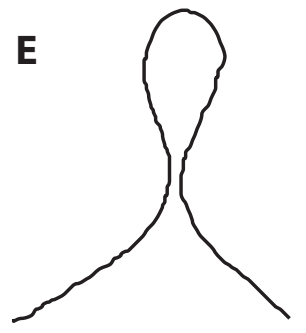

Figure 1. Curved tangible stimuli used in the experiments. The open convex curve (B) on the right was used in Experiment 1. Both curves (A and B) were tested in the second experiment. The closed convex curves ( $A$ and $C$ ) were used in Experiment 2. (D) Dimensions for the rotated closed forms in Experiment 3. (E) Flexible ruler bent in a pattern that was similar to that used to explain peak height and width judgments to the subjects. The subjects were told to judge the width of the stimulus at the widest point (here, at the base) and the peak height from the top of the pattern to the bottom.

to move the vertical ruler so that it extended out from the shelf as much as the pattern was tall. When judging the horizontal, they were to move the horizontal ruler so that it extended out from the vertical panel as much as the pattern was wide. The subjects were told to feel the ruler with their left hands after each ruler adjustment to be sure that they felt the extent and were not merely moving the ruler and making a judgment of this motion. In the touch groups, after blindfolding, but before data collection, the subjects were verbally guided to the tangible rulers, and their use was explained. Haptic subjects were not allowed to view the tangible rulers at any point, since we did not want to provide prior visual information about the possible range of sizes.

The stimuli were presented in ascending and descending size order; half of the subjects started with the largest stimuli, and half began with the smallest. However, the subjects judged the horizontal and vertical dimensions of each size of stimulus before exposure to another larger or smaller stimulus. For example, the subjects judged the horizontal and vertical of the 2.54-cm curve before exposure to the $5.08-\mathrm{cm}$ stimulus. Thus, each trial block consisted of a single presentation of all sizes of the stimuli, with judgments of the horizontal and vertical of each curve. After completion of a trial block using ascending size order, each of the subjects was exposed to a trial block with a descending size order. The order of presentation of ascending and descending sizes was balanced. Overall, each subject had two trial blocks with ascending and two with descending size orders, but with an ascending and descending direction (or descending and ascending) in the first two trial blocks and an ascending and descending sequence in the third and fourth trial blocks (or vice versa). This method involving ascending and descending series was adopted in order to facilitate timely presentation of the stimuli and reduce the overall experimental duration. The experimental sessions lasted approximately $25-30 \mathrm{~min}$, with some individuals requiring 35 or $40 \mathrm{~min}$. It was thought that a completely randomized presentation format would increase variability, prolong the experimental sessions, and possibly induce fatigue in the subjects.

In addition, the haptic subjects were told to avoid resting their wrists on the table surface as they felt the curves. This instruction 

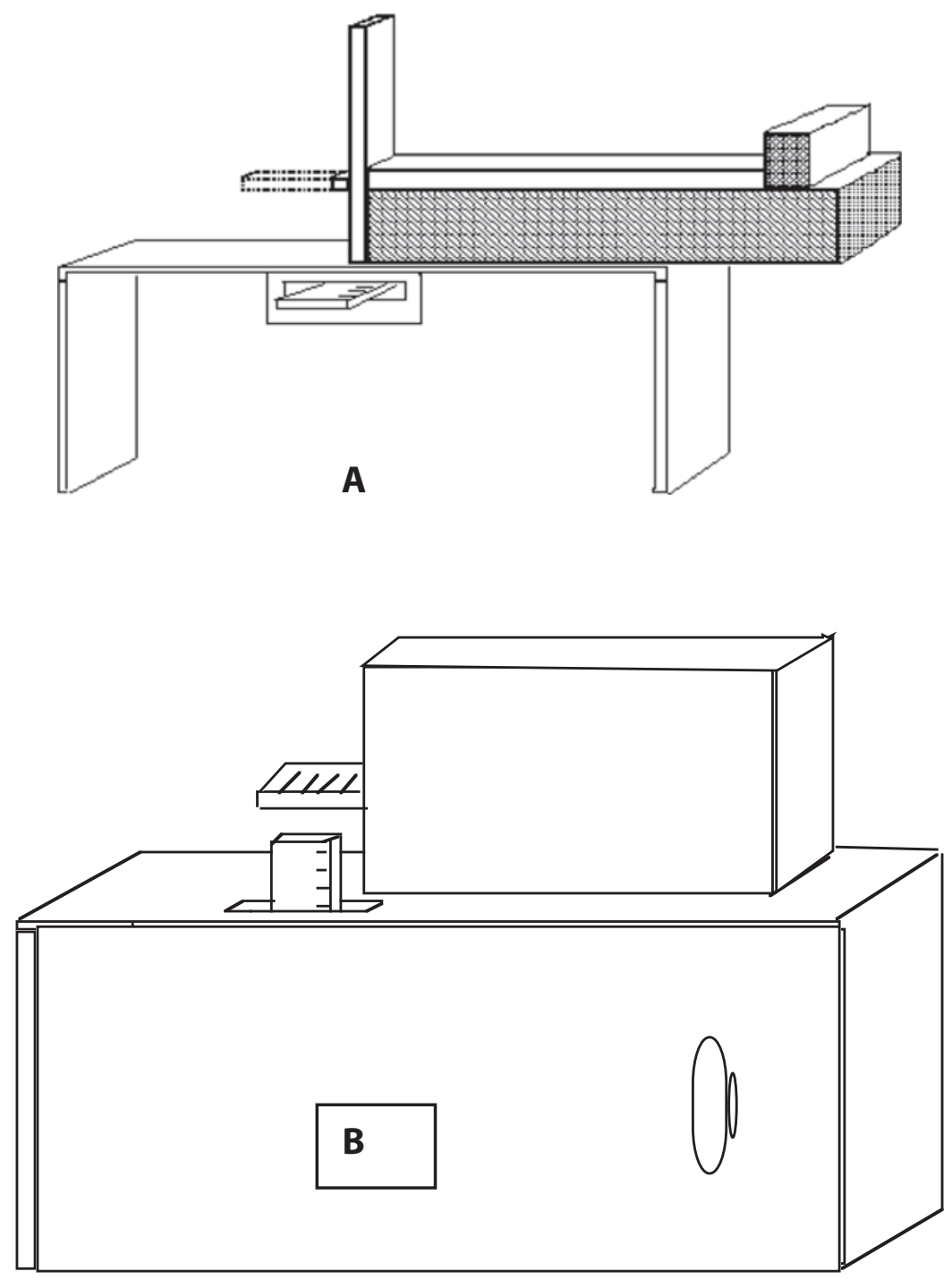

Figure 2. Tangible rulers used for size judgments. The top shows the arrangement when the stimuli were flat on the table surface. Stimuli were placed on the table surface (A) beneath the shelf that held the rulers and were at the body midline. The midpoint of each curve was at the midline, as was the front of the vertical panel from which the horizontal ruler extended. The bottom shows the arrangement used when the stimuli were frontal - that is, gravitationally vertical. Here, the stimuli were placed on the gravitationally vertical surface (B), with the midpoint of each stimulus at the body midline and secured by the clipboard.

was designed to induce whole arm motion, since this has been shown to be a contributor to the horizontal-vertical illusion with inverted-T and L shapes (Gentaz \& Hatwell, 2004; Heller et al., 1997; McFarland \& Soechting, 2007).

In the visual groups, the tangible rulers were replaced with maple wooden slats without markings. While seated subjects viewed the curves (but did not touch them), they made size judgments of the curves' horizontals and verticals. They did so by adjusting the visible wooden slats with their left hands so that they appeared visibly equal in extent to the dimension that they judged using vision. After each horizontal or vertical estimate, the entire apparatus was covered by a sheet of foam board, and the judgment (wooden slat extent) was measured by the experimenter, using a ruler with visible markings. The subjects viewed the curves again, and then judged the other dimension, horizontal or vertical, of the curve. The subjects were not allowed to see the measurements that corresponded to their size estimates. The method for vision was comparable to that for touch in all important respects, other than use of vision of the curves and the availability of sight of the hand and of the wooden slats during size judgments. The subjects were allowed continual sight of the curves while they made their size judgments by manipulating the visible wooden slats.

\section{Results and Discussion}

The subjects overestimated the verticals of the curves, as compared with their horizontals, in touch and vision. Percentage of illusions could be computed by subtracting horizontal judgments from verticals and dividing by the horizontal judgment. However, an alternative, more con- 


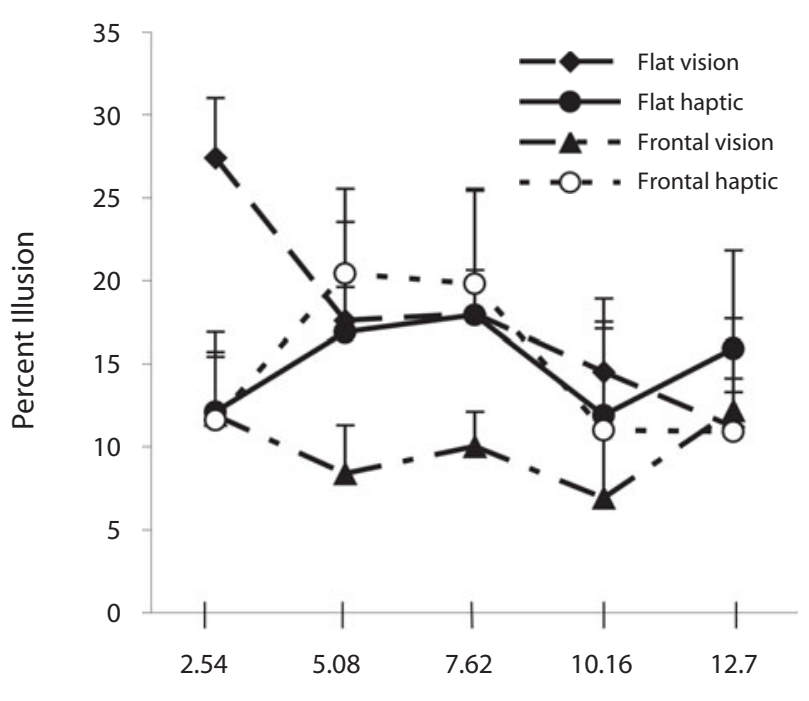

Size $(\mathrm{cm})$

Figure 3. The results of Experiment 1 collapsed over trials for the visual and haptic groups. The data are percentage-of-illusion scores derived from subtracting the judged horizontal from the judged vertical $\times 2$, and dividing by the sum of the judged horizontal and vertical. These data represent the relative size of the illusion and show the relative overestimation of the vertical, as compared with horizontal judgments. The error bars show the standard errors (for the top half).

servative method was adopted. The data were transformed by subtracting the judged horizontal of each stimulus from the judged vertical $\times 2$, and dividing by the sum of the judged horizontal and vertical. The results are summarized in Figure 3 and show a strong horizontal-vertical illusion with tangible and visible curves. An ANOVA was run on the transformed percentage of illusion scores. Mean percentage of illusion was slightly larger for the flat group $(M=$ $16.4 \%)$ than for the frontal group $(M=12.3 \%)$, but this effect was nonsignificant $[F(1,44)=1.1, p=.3]$. In addition, the illusion was similar in strength in vision $(M=13.8 \%)$ and in touch $(M=14.8 \%)[F(1,44)=0.07, p=.79]$. There was a significant interaction between modality and size $\left[F(4,176)=2.89, p=.0239, \eta_{\mathrm{p}}^{2}=.06\right]$. Tests of the simple effects of the interaction showed that the effect of modality was nonsignificant for all sizes of stimuli (all $p \mathrm{~s}>.10$ ) but that the effect of size was significant for both vision and touch $(p<.05)$. There was also a significant triple interaction between position, modality, and trials $[F(3,132)=$ $\left.4.76, p<.01, \eta_{\mathrm{p}}^{2}=.098\right]$. The illusion appeared to diminish in strength over trials for vision in the frontal group, but not any of the others. None of the other main effects or interactions was significant (all $p \mathrm{~s}>$.063).

A second ANOVA was conducted on size estimates. For this analysis, the data were transformed by adding the horizontal and vertical judgments and dividing by 2 . The effect of position failed to reach significance $[F(1,44)=2.99$, $p=.09]$, but the effect of modality was highly significant $\left[F(1,44)=55.39, p<.001, \eta_{\mathrm{p}}^{2}=.56\right]$, with much larger size judgments for vision $(M=9.0 \mathrm{~cm})$ than for touch $(M=$ $6.4 \mathrm{~cm})$. The illusion was not limited to one position, flat or frontal. This suggests that other processes, in addition to radial scanning, probably contributed to the illusion.

The elimination of radial scanning did not significantly reduce the overall potency of the haptic illusion in the present experiment, and this was not expected. Moreover, size estimates were closer to the true size when the stimuli were flat on the table surface. It was thought that the overestimation of verticals, as compared with horizontals, might be derived mainly from radial scanning of the sides of the curves. If the illusory overestimation of verticals versus horizontals with curves follows identical causal principles as one finds for $\mathrm{L}$ shapes and inverted-T shapes, one might have expected a significant interaction effect between group (position) and orientation. Note, however, that there was a significant three-way interaction between trials, position, and the illusion. An examination of the means indicated that the illusion diminished in strength over trials for vision in the frontal position but that the reverse occurred when the stimuli were flat. Haptics showed an increase in illusion strength over trials for the frontal stimuli but little change over trials when the stimuli were flat. Also, the illusion that one finds with inverted-T shapes has two causes, radial scanning and bisection. A number of causal factors could also be at work in the curvature illusion.

One might wonder whether there was some sort of substantial bias that could have been introduced by the tangible rulers and whether this contributed to the haptic illusion. However, the methods used are not all that different from those used in other research for the study of illusions (Amazeen \& DaSilva, 2005) and have advantages over the use of a fingers posture for size estimates (e.g., Heller et al., 1997; Heller, Joyner, \& Dan-Fodio, 1993; Smeets, Brenner, de Grave, \& Cuijpers, 2002). The rulers were in different spatial locations than the extents being judged, and conceivably, this could prompt systematic errors. This is properly the subject of future research in this area. Also, it is possible that the illusion would be diminished if the stimuli were felt with one hand-for example, the right hand in the right hemispace. This would align the stimulus pattern with the shoulder, and there is some evidence that the haptic egocenter changes with the task (see Soechting \& Flanders, 1993; Soechting, Tillery, \& Flanders, 1990). Thus, alignment with a shoulder-centered frame of reference may reduce perceptual errors and diminish the illusion. Heller et al. (1997) used very different methods but did report a diminished horizontal-vertical illusion for inverted-T and L stimuli when they were felt and judged using the right hand in the right hemispace. The possible importance of stimulus alignment with the shoulder should be examined in future research, and there is a great deal more that should be attempted in this area.

One might speculate about whether the illusion can be explained in terms of a bias introduced by using ascending and descending sequences. It is possible that the illusion could be limited to ascending series. To rule out this possibility, the flat group data of Experiment 1 were sorted by ascending and descending series. The illusion was stronger in the ascending condition $(M=15.8 \%)$ than in the descending condition $(M=12.4 \%)$, but ANOVAs on size estimates showed a nonsignificant effect of direc- 
tion (ascending vs. descending) $[F(1,11)=1.26, p>.25]$, and the interaction between orientation and direction also failed to reach significance $(F<1)$. An ANOVA on percentage of illusion also yielded a nonsignificant effect of ascending versus descending direction $(F<1)$. Thus, the illusion is not limited to one sort of sequence.

There is another interpretation of the results that must be considered. Lederman, Klatzky, and Barber (1985) found evidence for a movement-based heuristic in judgments of the Euclidean distance between the endpoints of irregular curves. This is the idea that the movements that one makes can influence judgments of extent. Increases in path length prompted increases in the judged distance between endpoints (also see Faineteau, Gentaz, \& Viviani, 2003, 2005). It is possible that the subjects overestimated the verticals of the curves in Experiment 1-in part, because of increases in movement extent while scanning the curved pathway. This could represent a possible cause of one component of the illusion involving overestimation of verticals. Furthermore, Heller et al. (2008) varied curve verticals and asked subjects to make diameter judgments. Experiment 5 of that study also manipulated the number of times that the subjects traced the stimuli $(1 \times, 9 \times$, or unlimited traces). When the subjects were limited to a single trace, diameter judgments increased as a function of the vertical ratio, and this is consistent with the idea of a movement-based heuristics explanation. However, this result was not obtained when the subjects traced the stimuli $9 \times$ or in the unlimited traces group. Nonetheless, Heller et al. (2008) found differences in path completion as a function of the number of traces, with smaller diameter judgments for nine traces or unlimited numbers of traces. Thus, path completion length was affected substantially by tracing. Also, they reported that the illusion involved underestimation of horizontals and overestimation of verticals. Perhaps tracing the peak of the curves serves to functionally bisect them. The change in scanning direction may accomplish this. The bisection effect may be analogous to what happens with inverted-T shapes. More empirical work will be needed in this area to clarify the possible role of movement-based heuristics, since multiple factors are likely at work. This issue is taken up again at a later point in this article.

It is important that radial scanning was eliminated in Experiment 1; yet overestimation of verticals was still obtained with curves. This is counter to the earlier reports of Day and Avery (1970) and Heller et al. (2003). Radial scanning plays a key role with inverted-T figures, and researchers have linked overestimation of radial lines to differences in scanning rate and inertia (see McFarland \& Soechting, 2007). Radial movements are slower and require more effort. However, differences in movement velocity did not have an impact on curvature (Soechting \& Poizner, 2005).

\section{EXPERIMENT 2 \\ The Effect of Pattern Rotation on the Haptic and Visual Horizontal-Vertical Curvature Illusion}

Experiment 1 yielded ambiguous evidence for the impact of radial scanning on the haptic horizontal-vertical curvature illusion. However, it is important to note that, although tracing the sides of a convex pattern yields arm motions that converge toward the body, finger movements are not perfectly radial. In the second experiment, we sought to further examine the impact of relevant factors by rotating the curves $90^{\circ}$ to the right. The effect of the positive rotation was to prompt radial scanning of the bases of curves and eliminate most of this forward-and-back arm motion during scanning for the rotated curved lines themselves (see also Künnapas, 1955; Tedford \& Tudor, 1969). In vision, rotation of an inverted-T shape makes the continuous line horizontal and yields overestimation of the horizontal.

\section{Method}

Subjects. The blindfolded subjects were 15 (11 females and 4 males) naive undergraduate student volunteers who received course credit for their participation. One of the females was lefthanded, but all of the other subjects were strongly right-handed. An additional 15 naive subjects used vision ( 8 females and 7 males).

Stimuli and Apparatus. The stimuli were convex curves with a baseline connecting the endpoints (see Figure 1A). The tangible curves were the same sizes as those in Experiment 1. The stimuli were always flat on the table and were either upright (as in Figure 1A) or rotated $+90^{\circ}$ (Figures $1 \mathrm{C}$ and 1D). The tangible rulers are shown in Figure 2 (top). In the visual group, the rulers were replaced by wooden slats without markings (as in the visual group in Experiment 1).

Design and Procedure. The experiment was a between-within design, with independent groups for modality (vision vs. touch); repeated measures were taken on rotation of the stimuli (upright or $+90^{\circ}$ ), horizontal versus vertical judgments of extent, size (5), and trials (2). The stimuli were blocked by rotation, and the subjects were randomly assigned to starting with the upright or rotated stimuli; they then completed the experiment with the other rotation condition. In touch, 8 of the subjects started with the patterns upright, and 7 began the experiment with the stimuli rotated $+90^{\circ}$. In most other respects, the method was similar to that in the earlier experiment. They were instructed when the position of the stimuli changed - that is, from upright to rotated or vice versa. The subjects always used the horizontal ruler for width (horizontal) judgments and the radial/ vertical ruler for height (vertical) judgments (see Figures 1 and 2). The subjects were told to judge the heights of the curved stimuli and their widths at their greatest extent.

The procedure was identical for vision, but with a few modifications. After each size estimate, a large sheet of foam board was interposed between the subject and the unmarked wooden slats, so that the experimenter could measure size estimates with a ruler. The wooden slats were then returned to the starting position prior to the next judgment (horizontal or vertical).

\section{Results and Discussion}

A strong illusion was obtained (see Table 1), but the illusion was stronger for touch $(M=16.7 \%)$ than for vision $(M=6.7 \%)$. Furthermore, the illusion was far stronger when the patterns were upright $(M=15.9 \%)$ than when rotated $(M=7.4 \%)$. An ANOVA was performed on percentage of illusion scores, computed as in Experiment 1. The effect of modality was significant $[F(1,29)=5.4, p=$ $\left..03, \eta_{\mathrm{p}}^{2}=.156\right]$, as was the effect of rotation $[F(1,29)=$ $\left.10.8, p<.01, \eta_{\mathrm{p}}^{2}=.11\right]$, but the interaction between modality and rotation failed to reach significance $[F(1,29)=$ $3.4, p=.074]$. None of the other main effects or interactions reached significance (all $p \mathrm{~s}>.07$ ).

To further clarify possible differences between vision and touch, separate ANOVAs were run on the size judg- 
Table 1

Mean Judgments of Extent, Standard Deviations (SDs), and Mean Percentages of Illusion for Tangible Convex Curves That Were Upright or Rotated $+90^{\circ}$ in Experiment 2

\begin{tabular}{|c|c|c|c|c|}
\hline \multirow[b]{3}{*}{ True Size } & \multicolumn{4}{|c|}{ Rotation } \\
\hline & \multicolumn{2}{|c|}{ Upright } & \multicolumn{2}{|c|}{ Rotated $+90^{\circ}$} \\
\hline & $\mathrm{H}$ & V & $\mathrm{H}$ & V \\
\hline & \multicolumn{2}{|c|}{ Touch } & & \\
\hline \multicolumn{5}{|l|}{$2.5 \mathrm{~cm}$} \\
\hline$M$ Judgment & 2.30 & 2.90 & 2.47 & 2.92 \\
\hline$S D$ & 0.47 & 0.73 & 0.82 & 0.65 \\
\hline$\%$ Illusion & \multicolumn{2}{|c|}{23.1} & \multicolumn{2}{|c|}{16.7} \\
\hline \multicolumn{5}{|l|}{$5.1 \mathrm{~cm}$} \\
\hline$M$ Judgment & 4.00 & 4.93 & 4.42 & 5.07 \\
\hline$S D$ & 0.90 & 1.20 & 1.41 & 1.06 \\
\hline \% Illusion & \multicolumn{2}{|c|}{20.8} & \multicolumn{2}{|c|}{13.7} \\
\hline \multicolumn{5}{|l|}{$7.6 \mathrm{~cm}$} \\
\hline$M$ Judgment & 5.93 & 7.5 & 6.74 & 7.17 \\
\hline$S D$ & 1.25 & 2.14 & 2.00 & 1.67 \\
\hline$\%$ Illusion & \multicolumn{2}{|c|}{23.4} & \multicolumn{2}{|c|}{6.2} \\
\hline \multicolumn{5}{|l|}{$10.2 \mathrm{~cm}$} \\
\hline$M$ Judgment & 8.15 & 9.45 & 8.61 & 10.06 \\
\hline$S D$ & 1.93 & 2.59 & 2.40 & 2.68 \\
\hline$\%$ Illusion & \multicolumn{2}{|c|}{14.8} & \multicolumn{2}{|c|}{15.5} \\
\hline \multicolumn{5}{|l|}{$12.7 \mathrm{~cm}$} \\
\hline$M$ Judgment & 9.65 & 11.70 & 10.55 & 12.26 \\
\hline$S D$ & 2.22 & 3.21 & 2.64 & 2.44 \\
\hline \multirow[t]{2}{*}{$\%$ Illusion } & \multicolumn{2}{|c|}{19.2} & \multicolumn{2}{|c|}{15.0} \\
\hline & \multicolumn{2}{|c|}{ Vision } & & \\
\hline \multicolumn{5}{|l|}{$2.5 \mathrm{~cm}$} \\
\hline$M$ Judgment & 2.78 & 3.59 & 3.01 & 3.12 \\
\hline$S D$ & 0.37 & 1.30 & 0.38 & 0.72 \\
\hline$\%$ Illusion & \multicolumn{2}{|c|}{25.4} & & \\
\hline $5.1 \mathrm{~cm}$ & & & & \\
\hline$M$ Judgment & 5.34 & 6.20 & 5.63 & 5.75 \\
\hline$S D$ & 0.61 & 1.01 & 0.69 & 1.23 \\
\hline$\%$ Illusion & & & & \\
\hline $7.6 \mathrm{~cm}$ & & & & \\
\hline$M$ Judgment & 7.76 & 8.85 & 8.18 & 8.17 \\
\hline$S D$ & 0.60 & 1.52 & 1.08 & 1.49 \\
\hline$\%$ Illusion & & & & \\
\hline $10.2 \mathrm{~cm}$ & & & & \\
\hline$M$ Judgment & 10.04 & 11.13 & 10.42 & 10.48 \\
\hline$S D$ & 0.69 & 1.78 & 1.36 & 1.55 \\
\hline$\%$ Illusion & & & & \\
\hline $12.7 \mathrm{~cm}$ & & & & \\
\hline$M$ Judgment & 12.49 & 14.06 & 12.94 & 12.62 \\
\hline$S D$ & 1.11 & 2.74 & 1.65 & 1.85 \\
\hline$\%$ Illusion & & & & \\
\hline
\end{tabular}

Note-Mean size judgments were computed with the data collapsed over trials. H, horizontal; V, vertical. Percentage-of-illusion scores = $2(\mathrm{~V}-\mathrm{H}) /(\mathrm{H}+\mathrm{V})$. A positive illusion is shown by an overestimation of the verticals.

ments for the bases and the curves. An ANOVA on the size judgments for the bases showed a significant effect of modality $\left[F(1,29)=8.8, p<.01, \eta_{\mathrm{p}}^{2}=.233\right]$ and a highly significant effect of rotation $[F(1,29)=24.3, p<.001$, $\left.\eta_{\mathrm{p}}^{2}=.455\right]$. The interaction between modality and orientation was also significant $\left[F(1,29)=9.9, p<.01, \eta_{\mathrm{p}}^{2}=\right.$ $.254]$. An examination of Table 1 shows larger size judg- ments for the bases when they were vertical for touch, but the difference between comparable judgments was much smaller for vision. Recall that when the curves are rotated, the horizontal base for the upright curve becomes a vertical straight line for the rotation condition. The larger effect for touch than for vision is probably a consequence of radial scanning of the baselines for the rotated stimuli. Radialtangential scanning effects contributed to the stronger illusory overestimation of the bases for touch than for vision. An ANOVA on the size estimates for the curves yielded a significant effect of modality $[F(1,29)=8.9, p<.01$, $\left.\eta_{\mathrm{p}}^{2}=.24\right]$ and a significant effect of rotation $[F(1,29)=$ $\left.8.4, p<.01, \eta_{\mathrm{p}}^{2}=.23\right]$ but a nonsignificant interaction $(F<1)$. The curves were judged as larger overall when upright $(M=8.0 \mathrm{~cm})$ than when rotated $(M=7.3 \mathrm{~cm})$.

Although the illusion occurs in both modalities, it was weaker in the visual modality. Moreover, it was affected differently by rotation. When the curves were rotated, vision showed an especially weak illusion, with little difference between the judgments of extent of the vertical base and horizontal curve (see Table 1). Touch seemed susceptible to the effect of radial scanning, with substantial overestimation of the vertical base in the rotation condition. Thus, the illusion was found in both vision and touch, but perhaps for different reasons. Both senses are influenced by the curved configuration and show the horizontal-vertical illusion, but in touch, radial scanning plays an important role. In sight, the visual tendency to overestimate verticals may compete with the visual overestimation of curves, even when rotated. Therefore, one sees a diminished or absent illusion when the curves are rotated in vision. Thus, the visual and haptic horizontalvertical illusion may also include a configural component. Stimulus size was also judged differently in the two modalities, and that is a concern for any position that assumes intersensory equivalence.

\section{EXPERIMENT 3 \\ Haptic Convex Curves With and Without a Base Line}

The haptic horizontal-vertical illusion with curves could derive, in part, from a higher level cognitive problem, rather than from a perceptual difficulty in judging extent. In the path completion problem, subjects feel a curve and then judge the distance between endpoints (see Faineteau et al., 2003, 2005; Lederman et al., 1985). They are not permitted to actually feel the distance between endpoints, and the subjects in Experiment 1 were prevented from scanning between endpoints. Thus, the subjects got to feel the peak height of the curve but must have mentally computed the distance between endpoints. They could do this in a number of ways, but the task in Experiment 1 did not allow the subjects the option of tracing the distance between the endpoints of the curves. Conceivably, the obtained overestimation of the vertical, as compared with the horizontal, could partially derive from this methodological constraint. Note, however, that the subjects did not directly feel the peak heights, since they were limited to tracing the curved path. The subjects 
might overestimate the vertical, relative to the horizontal, in open curves because of a contraction of the horizontal extent, analogous to closure.

Consequently, two groups of subjects in Experiment 3 felt convex curves whose verticals were equal to their horizontals. The subjects in one group had curves with a line across the base (see Figure 1A). The subjects in a second group did not have this baseline, as with the stimuli in Experiment 1 (Figure 1B). If relative underestimation of the horizontal derives from an inability of subjects to feel this horizontal extent, one might expect to find an elimination of this aspect of the horizontal-vertical illusion in Experiment 3. Moreover, if the illusion is dependent on a process analogous to closure, one would predict an elimination of the illusion in the third experiment for the stimuli with a baseline.

\section{Method}

Subjects. The blindfolded subjects were 24 (15 females and 9 males) undergraduate volunteers, all of whom were experimentally naive. All were right-handed, as in Experiment 1. The group with open convex curves had 12 subjects ( 8 females and 4 males), with another 12 subjects in the group with closed convex curves (7 females and 5 males).

Stimuli and Apparatus. The stimuli were similar to the convex curves in Experiment 1, but a second set of stimuli included a line connecting the endpoints of the curves at their bases (see Figure 1). Since all the stimuli were flat on the tabletop, the haptic rulers (see Figure 2) included a tangible ruler that was oriented horizontally (for judgments of the horizontals) and another one that was vertical but radially oriented (for judgments of verticals). In other respects, the stimuli and apparatus were similar to those in the first experiment.

Design and Procedure. The experiment was a between-within design, with independent groups for type of curve (open or closed), with repeated measures on orientation (vertical vs. horizontal), size (5), and trials (4). In most respects, the procedure was similar to that in Experiment 1. However, the subjects with the closed stimuli were permitted to trace the entire pattern. The subjects with the open convex curves were told that they should trace across the bottom of the curves. All the subjects were told that they could trace the stimuli in one direction, either clockwise or counterclockwise as they chose, but were not to trace repeatedly back and forth between the endpoints of the open curve or across the base of the closed forms. As in Experiment 1, the subjects were blindfolded throughout and were not given feedback on their judgments.

\section{Results and Discussion}

The results indicated a horizontal-vertical illusion, even when the subjects were permitted to trace between the endpoints at the base of convex curves. The illusion was larger for the open curves $(M=16.9 \%)$ than for the closed ones $(M=9.8 \%)$. However, an ANOVA was performed on percentage of illusion scores and indicated that the effect of group failed to reach significance $[F(2,22)=$ $1.4, p=.25]$. All of the other main effects and interactions were nonsignificant (all $p \mathrm{~s}>.10$ ), with the exception of an interaction between group, size, and trials $[F(12,264)=$ $\left.2.2, p=.014, \eta_{\mathrm{p}}^{2}=.09\right]$. This interaction effect size was rather small in magnitude and derived from a very small proportion of the variance.

A second ANOVA on size judgments transformed the data using the following formula: size $=(\mathrm{H}+\mathrm{V}) / 2$. The curves were judged as larger when closed $(M=8.0 \mathrm{~cm})$ than when open $(M=7.3 \mathrm{~cm})$, but this main effect of stimulus pattern was nonsignificant $(F<1)$. However, there was a significant effect of trials $[F(3,66)=5.4, p<$ $\left..01, \eta_{\mathrm{p}}^{2}=.196\right]$, owing to smaller judgments on the last trial $(M=7.3 \mathrm{~cm})$ than on the first $(M=8.1 \mathrm{~cm})$. There was also a significant interaction between group and trials $\left[F(3,66)=3.0, p<.05, \eta_{\mathrm{p}}^{2}=.12\right]$. Tests of the simple effects of this interaction showed that the effect of trials was limited to the open curves $(p<.01)$. The simple effect of trials failed to reach significance for the closed curves $(p>.50)$. The subjects judged the open curves as much smaller on the last trial $(M=6.54 \mathrm{~cm})$ than on the first trial $(M=7.93 \mathrm{~cm})$. These results are consistent with the idea that the nature of tracing can influence judged size (Lederman et al., 1985).

We wondered whether it mattered whether or not the subjects traced between the endpoints of open convex curves. Perception of the distance between the endpoints seemed more indirect than judgments of peak height. This led to a further ANOVA on size judgments that compared the group with open curves in the present experiment with the group from the first experiment, in which the curves were flat on the table surface and the subjects could not trace between endpoints. The results of this ANOVA on size judgments revealed a nonsignificant effect of group $(F=0.2)$, indicating that the illusion was not completely dependent on preventing the subjects from tracing between the endpoints of the curves. The illusion was similar in magnitude when the subjects could trace between endpoints $(M=16.9 \%)$ as when they were prohibited from doing so in Experiment $1(M=16.4 \%)$. Thus, the haptic illusion was not dependent on preventing the subjects from tracing between endpoints of the curves. Movement heuristics probably contributed to size judgments here and in Experiment 2 and, perhaps, to the nature of perceptual distortion. The contribution of movement heuristics to haptic judgments of extent is complex.

The illusion was weaker for the closed curves in the present experiment than in Experiment 2. This different outcome may be related to the larger number of trials in the present experiment and the different, repeated measures design in Experiment 2. Note that illusory misperception for open curves was nearly identical in the present experiment to that found in the earlier experiments in this report.

\section{EXPERIMENT 4 \\ Attenuation of the Horizontal-Vertical Haptic Illusion With Bimanual Touch at the Body Midline}

Conceivably, the presence of the horizontal-vertical illusion could derive from artificially constrained methods of feeling the stimuli. Thus, blind people have objected to being restricted to the use of a single finger when feeling patterns in earlier research (e.g., Heller et al., 2002). Some blind individuals compared feeling a pattern with a single finger with looking at something monocularly. Also, it is very possible that allowing subjects the option of feeling stimuli in any manner they wished would yield optimal haptic exploration methods. Tracing stimuli with 
a single finger may not be the "best" method for obtaining accurate size information; therefore, the subjects in Experiment 4 were allowed to use any combination of fingers that they desired. Note that, on some theoretical viewpoints, illusory misperception may derive from impoverished stimulation or poor "viewing" conditions (e.g., Gibson, 1979). Perhaps raised-line stimuli are impoverished (but see Heller et al., 2003; Kennedy, 1993), and this promotes illusory distortion.

There could be an even more important influence on the haptic horizontal-vertical curvature illusion, aside from the haptic exploration method. It is clear that bimanual exploration aids haptic judgments of symmetry (Ballesteros et al., 1997). This benefit of bimanual exploration probably derives from allowing the individual to code patterns with respect to the body midline and use the body as a frame of reference (e.g., Millar, 2006; Millar \& Al-Attar, 2002). Egocentric coding with respect to the midline can aid perceptual accuracy and has also been shown to help judgments of the vertical frame of reference (Heller, Calcaterra, Green, \& Barnette, 1999). Bimanual exploration also nearly eliminated the Müller-Lyer illusion for touch (Heller et al., 2005). The body is an "object" with a relatively familiar size. Thus, feeling the stimuli with two hands at the midline should allow subjects to feel their bodies with their elbows as they examine the stimuli. They could also use this body information as a frame of reference for size estimates for the felt lines. Furthermore, this ability to rest the two arms against the body could also allow more stable and secure scanning. If haptic coding with respect to a body frame of reference aids perceptual accuracy, the illusion should be diminished in Experiment 4.

\section{Method}

Subjects. There were two groups of undergraduate volunteers in the experiment, with 12 ( 9 females and 3 males) using bimanual free exploration. A second group ( 8 females and 4 males) used bimanual touch but were limited to the use of their index fingers. All were right-handed and experimentally naive and did not participate in the earlier experiments reported here.

Stimuli and Apparatus. The stimuli were open convex curves, with sizes like those in Experiment 1. The haptic ruler apparatus is shown in the top of Figure 2. As in Experiment 1, the stimuli were flat on the table surface.

Design and Procedure. The experiment was a between-within design, involving independent groups for mode of exploration (free or two index fingers), with repeated measures on orientation (horizontal vs. vertical), size (5), and trials (4). Each subject was exposed to four trial blocks, with the general method similar to that in Experiment 1 . The stimuli were flat on the table surface at the body midline (Figure 2). The subjects were instructed to use two hands to feel the patterns. In addition, free exploration subjects were told that they could feel the patterns any way that they wished; those in the other group were restricted to the use of two index fingers. However, they were limited to the use of the left hand for giving size estimates by manipulating the ruler. We limited the subjects to the use of one hand to minimize the potential problem of a use of two hands to "move measurements" from the stimuli themselves to the rulers. Since they were limited to one hand, they would be unable to use one hand as a "ruler" or caliper and then transfer the measurement to the wooden ruler apparatus. That is, we wished to prevent the subjects from laying a finger/hand along an extent and then "marking" the extent by holding the index finger of the other hand at that location on the body surface. We were concerned that the subjects might then attempt to transfer this mechanical measurement to the ruler apparatus. Half of the subjects made judgments of the vertical first and then judged the horizontal; half used the reverse sequence. The horizontal ruler was used for judgments of the horizontal, and the radial (vertical) ruler was used for judgments of the vertical.

\section{Results and Discussion}

The results are shown in Figure 4 and indicate that the haptic illusion was virtually eliminated when stimuli were explored bimanually at the body midline using free exploration $(M$ illusion $=2.3 \%)$. The data were transformed as in Experiment 1. The illusion was considerably larger when the subjects were restricted to the use of their two index fingers ( $M$ illusion $=17.2 \%)$. An ANOVA on these transformed percentage of illusion scores revealed a significant main effect of manner of exploration $[F(1,22)=$ $10.48, p<.01, \eta_{\mathrm{p}}^{2}=.32$ ], owing to a much larger illusion when the subjects were limited to the use of two index fingers. However, there was a significant interaction between group and size $\left[F(4,88)=5.1, p=.001, \eta_{\mathrm{p}}^{2}=.19\right]$. Whereas the illusion was relatively small overall for the free exploration group, the story was very different for the group limited to the use of two fingers. Here, with the use of two index fingers, the illusion was much larger for smaller stimuli and diminished as the stimuli increased in size. Tests of the simple effects of the interaction between group and size indicated that the effects of group were all significant for 2.54- to 10.2 -cm stimuli (all $p \mathrm{~s}<.05$ ), but not for the largest stimuli $(F<1, p>$.7). In free exploration conditions, tracing may become more likely for the largest stimuli, especially for individuals with smaller hands. Hence, we find the emergence of the illusion for the largest $12.7-\mathrm{cm}$ stimuli. The other main effects and interactions failed to reach significance (all $p \mathrm{~s}>.11$ ).

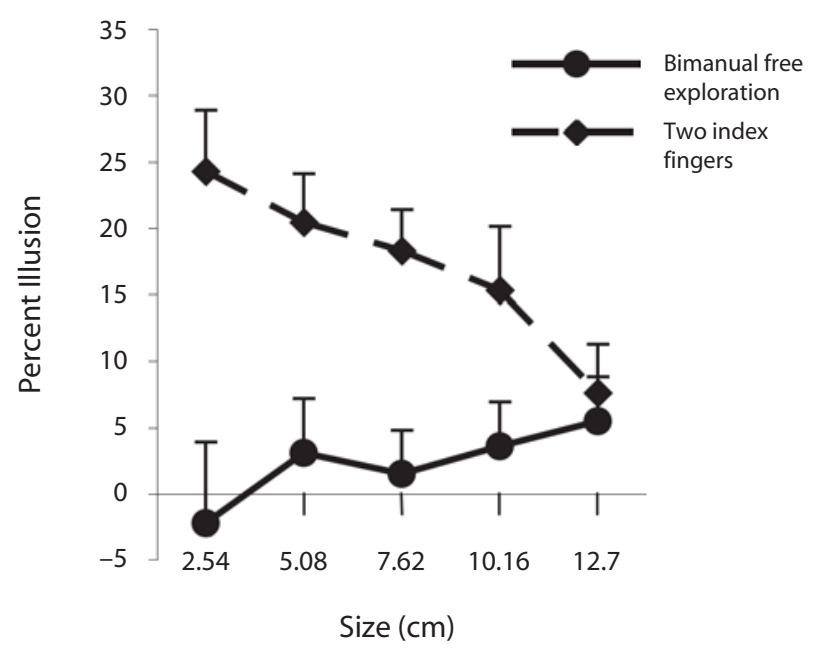

Figure 4. Percentage of illusion scores for the bimanual free exploration and bimanual two index finger groups in Experiment 4. Percent illusion was computed by subtracting the judged horizontal from the judged vertical $\times 2$, and dividing by the sum of the judged horizontal and vertical. Percentage of illusion is a measure of the magnitude of the illusion. 
Perhaps the hands "got in each other's way" in the two index fingers group for the smaller stimuli, and this impaired accurate perception. Thus, if one feels the horizontal extent with two index fingers of one hand, it may be difficult to get an accurate idea of extent, because each finger pad takes up a rather large proportion of the smaller lines that are felt.

A second ANOVA was conducted on transformed size estimates, as in Experiment 1. The stimuli were judged as larger using free exploration $(M=8.4)$ than when explored with two index fingers $(M=7.0)$, and this difference was highly significant $[F(1,22)=20.9, p<.001$, $\left.\eta_{\mathrm{p}}^{2}=.49\right]$. Whereas the effect of size was significant $\left[F(4,88)=1,097.9, p<.0001, \eta_{\mathrm{p}}^{2}=.98\right]$, the interaction between size and group was also significant $[F(4,88)=$ $\left.10.0, p<.001, \eta_{\mathrm{p}}^{2}=.31\right]$. Tests of simple effects showed that the effect of group failed to reach significance for the smallest, $2.54-\mathrm{cm}$ stimuli $(F<1)$, but all of the other tests of the simple effects of group were significant for the other sizes (all $p \mathrm{~s}<.01$ ).

The results were somewhat different from those previously reported for the Müller-Lyer illusion (Heller et al., 2005). In that earlier study, the use of two index fingers almost eliminated the Müller-Lyer illusion. The problems posed by Müller-Lyer stimuli are somewhat different from the horizontal-vertical curvature illusion. The horizontalvertical illusion is magnified by tracing with the index fingers, but we do not know whether bimanual tracing will aid perception with inverted-T configurations. The haptic Müller-Lyer illusion is partially derived from an inability to feel the extent of the line shaft when surrounded by wings-in arrows, perhaps because of sensory inhibition. The wings-in arrows at the end of the line shaft impeded the subjects' ability to feel the ends of the line, but only when explored by a single index finger. Thus, the subjects were unlikely to go beyond the endpoint of a line when they could no longer feel it. They then did not feel the full line extent in the Müller-Lyer stimuli. The use of two index fingers in that configuration prompted larger ranging exploration that included the endpoints. The present results with curves implicate the importance of combining free exploration with bimanual exploration, and the relevant causal mechanisms may be different.

The use of free exploration and bimanual exploration of the stimuli at the midline practically eliminated the horizontal-vertical illusion with curves. These results indicate that haptic illusions can be substantially attenuated if subjects are encouraged to adopt optimal methods for feeling the stimuli and they are relatively small.

It appears likely that the advantage of bimanual exploration accrued from allowing subjects the ability to make use of coding with respect to the body midline and with respect to the body itself (see Ballesteros et al., 1997; Ballesteros, Millar, \& Reales, 1998; Millar \& Al-Attar, 2000). Presumably, the body is a known metric, and relating bimanual tracing to this familiar size can be used to aid size estimates. In addition, one hand can serve as a spatial anchor for the other. If one explores lines with a single index finger or a single hand, the effect may be analogous to looking at a point light source in a darkened room. However, it is still possible that the benefits of bimanual exploration depended on allowing subjects free exploration, rather than allowing them to feel stimuli at the body midline. Thus, many subjects were observed using strategies that were not available to them in the earlier experiments reported in this article. Some subjects used their index finger and thumb in an L shape to try to "measure" the vertical. Other subjects tried to use pairs of fingers to engage in a grasp of the extents that they judged and then tried to transfer this to the ruler. Another strategy involved the use of multiple fingers to "measure" extent. The use of two hands also allowed the subjects to feel the horizontal and vertical extents simultaneously. According to Appelle (1991), unrestricted active exploration yields more accurate judgments of extent. Note that these strategies were not available to the subjects restricted to the use of the two index fingers alone, and these results were not identical to the effect of bimanual scanning of the tactual Müller-Lyer illusion. In the present instance, it is likely that attenuation of the horizontalvertical curvature illusion for smaller stimuli derived from the use of bimanual exploration at the midline, in combination with free exploration methods.

\section{EXPERIMENT 5 Left Versus Right Hand and Free Exploration at the Midline}

The results of Experiment 4 could have derived from free exploration at the body midline or from bimanual free exploration. Thus, it is still possible that the attenuation of the illusion could have derived from free haptic exploration, rather than from bimanual exploration at the midline. This issue was the motivation for Experiment 5, where subjects freely explored stimuli with their left or right hands at the midline. Presumably, bimanual exploration at the midline allows coding in terms of a body-centered frame of reference, which could aid haptics (see Ballesteros et al., 1998; Millar, 2006). Consequently, the subjects in Experiment 5 were restricted to the use of a single hand to feel the stimuli, using either their left or right hands. They used their other hands for size estimates with the tangible rulers. If free exploration alone can explain the benefits of bimanual examination in Experiment 4, the horizontal-vertical illusion should not be found in Experiment 5. Moreover, illusions may be influenced by hand, since the right hand sends pattern information to the left brain. The left brain may excel for verbal processing, but not spatial analysis (see Heller, Rogers, \& Perry, 1990). Conceivably, the use of the right hand could magnify perceptual error and increase the strength of haptic illusions (see Heller et al., 1993).

\section{Method}

Subjects. There were two groups of experimentally naive, righthanded, blindfolded subjects, with 12 per group, for a total $N$ of 24 . They included those individuals who used their right hand for feeling the patterns and their left hands for the rulers ( 8 females and 4 males), and others who used their left hands to feel the patterns and their right hands to adjust the rulers ( 9 females and 3 males).

Stimuli and Apparatus. The tangible stimuli were like those in Experiment 1 and were placed on the table surface at the midline, 
directly beneath the shelf that held the tangible rulers. The tangible rulers were the same, except that the direction of the rulers differed for the two groups. When the subjects felt the stimuli with their right hands, the ruler faced to the left, as in the earlier experiments. However, when the ruler was adjusted by the right hand and the stimuli were felt by the left hand, the ruler faced to the right. This method was adopted to avoid forcing the subjects to cross the body midline when feeling the patterns with the left hand and making size estimates with the right hand.

Design and Procedure. The experiment was a between-within design, with independent groups for the hand used to feel the curves (right vs. left). Repeated measures were taken on orientation (horizontal vs. vertical), size (5), and trials (4). In many respects, the method was identical to that in Experiment 4, except that the subjects were restricted to using one hand to feel the stimuli, and ruler direction changed with hand. They used either their left or right hand to feel the stimuli and used the opposite hand for size estimates.

\section{Results and Discussion}

The results are summarized in Figures 5 and 6. The illusion was somewhat stronger when stimuli were felt with the right hand $(M=15.22 \%)$ than when felt with the left hand $(M=8.87 \%)$. This difference between the hands appears large. However, an ANOVA on transformed percentage of illusion scores showed that the effect of group was nonsignificant $[F(1,22)=2.96, p=.10]$, despite a larger illusion for feeling the patterns with the right hand. A mixed-factor ANOVA on transformed size estimates also revealed that the main effect of hand failed to reach significance $[F(1,22)=2.3, p=.144]$. There was a significant main effect of trials $\left[F(3,66)=3.4, p=.02, \eta_{\mathrm{p}}^{2}=\right.$ $.133]$ and a significant effect of size $[F(4,88)=1,149.9$, $\left.p<.0001, \eta_{\mathrm{p}}^{2}=.98\right]$. However, the interaction between size and trials was significant $[F(12,264)=1.9, p=.03$, $\left.\eta_{\mathrm{p}}^{2}=.081\right]$. The interaction involved a significant simple effect of trials for the largest, 12.54-cm stimuli $(p<.01)$, but not for the smaller stimuli (all $p \mathrm{~s}>.16$ ). Overall,

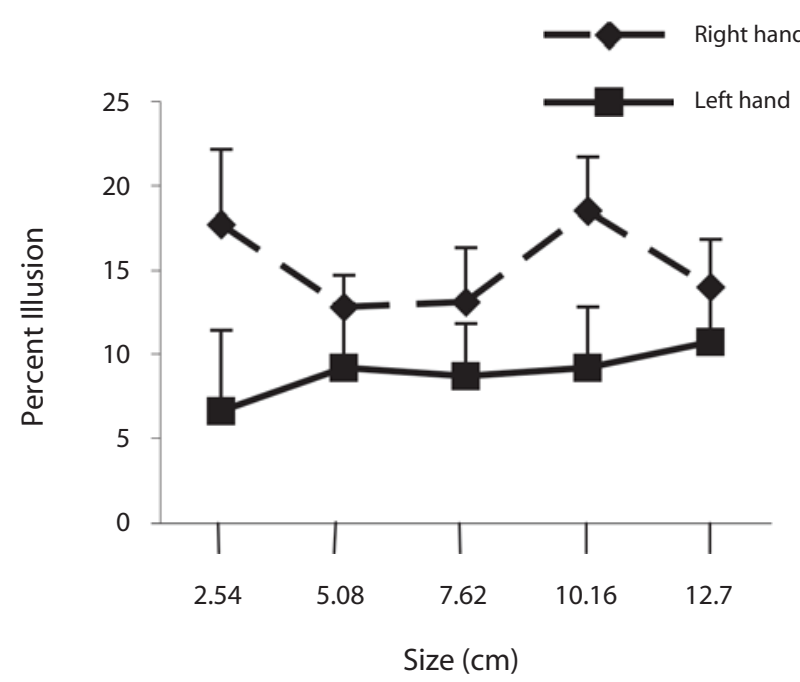

Figure 5. Mean percentage-of-illusion data for Experiment 5. We computed percentage of illusion by subtracting the judged horizontal from the judged vertical $\times 2$, and dividing by the sum of the judged horizontal and vertical. This provides an estimate of illusion strength. The subjects in Experiment 5 used a single hand, their left or right hand, to feel the stimuli at the midline.

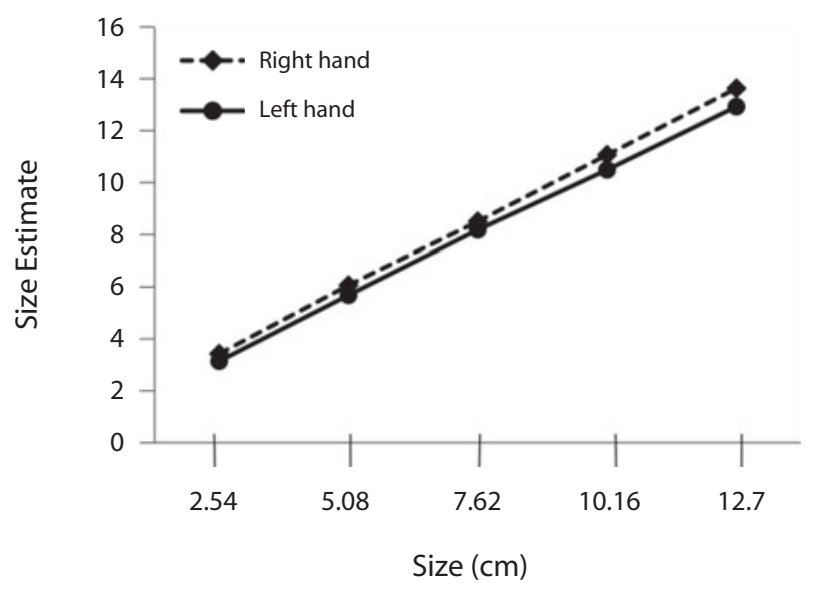

Figure 6. Size estimates for Experiment 5. These size estimates were obtained by data transformation by adding the horizontal and vertical judgments and dividing by 2 .

the subjects showed a slight overestimation of size (see Figure 6).

Further research is needed in this area, since a larger illusion with the use of the right hand would be consistent with the notion that the right brain is better organized for spatial perception. On this view, one would expect smaller errors with the left hand, since illusions of extent represent spatial errors. However, effects of hand and hemispace have not been entirely reliable in the haptic illusion literature (see Heller et al., 1997; Summers \& Lederman, 1990). Future research should be directed toward an examination of possible hand effects on the illusion.

The illusion was found when the subjects were limited to the use of a single hand in Experiment 5, even when they were permitted to feel the stimuli in any manner that they wished. This means that the results of Experiment 4 are not explainable solely in terms of free exploration and must be related to the use of two hands, in combination with free exploration for feeling the stimuli at the midline. The results are consistent with the idea that bimanual exploration at the body midline facilitates coding in terms of a bodily frame of reference (see Ballesteros et al., 1998; Millar, 2006). When one uses two hands to feel stimuli, one's arms may be in contact with the sides of the body. This provides an egocentric frame of reference, and body scale, a known and familiar metric, can be used to map the extents felt by the hands to a familiar scheme. Note that the results differed from those reported by Millar and AlAttar (2000), since they showed that exploration with the right index finger at the midline practically eliminated the illusion with an $\mathrm{L}$ shape. The curved configuration clearly yields a strong horizontal-vertical illusion that is difficult to eliminate in touch.

\section{EXPERIMENT 6 Bimanual Exploration in the Left or Right Hemispace}

The results of bimanual exploration at the body midline in Experiment 4 were striking but could be explained in 
a number of ways. The benefits of bimanual exploration could be linked to feeling patterns with both hands at the body midline, where the subjects could readily use their bodies as a scale for calibrating haptic size. Alternatively, the subjects could have been using their hands as a frame of reference. One hand may help individuals locate the other hand in space. On the former interpretation, the haptic horizontal-vertical curvature illusion should emerge when subjects feel patterns with both hands in the left or right hemispace. Two groups of naive subjects felt patterns in the left or right hemispace in Experiment 6. If bimanual free exploration alone could explain the attenuation of the illusion in Experiment 4, subjects should not show an illusion in this experiment. However, if exploration at the body midline were critical, one would expect to find a potent horizontal-vertical curvature illusion in Experiment 6.

\section{Method}

Subjects. There were two groups of experimentally naive blindfolded subjects with 14 per group (total $N=28 ; 11$ females and 3 males per group). All the subjects were right-handed.

Stimuli and Apparatus. The stimuli and apparatus were identical to those in Experiment 4. The midpoint of each stimulus was placed $34.5 \mathrm{~cm}$ to the left or right of the body midline, for the lefthemispace and right-hemispace groups. The horizontal ruler faced to the left, as in Experiment 4.

Design and Procedure. The experiment was a between-within design, with independent groups for hemispace (right vs. left) and with repeated measures for orientation (2), size (5), and trials (4). The procedure was similar to that in Experiment 4. The subjects were encouraged to use both hands to feel the stimuli whenever possible. In addition, they were told that they could always feel the stimuli again, since they were making size estimates with the tangible rulers. As in Experiment 4, they were limited to the use of the left hand for ruler adjustment, and ruler position was not altered for the two hemispace groups.

\section{Results and Discussion}

The results showed a horizontal-vertical illusion, with overestimation of verticals $(M=8.1 \mathrm{~cm})$, as compared with horizontals $(M=7.4 \mathrm{~cm}$; see Figure $7 ; M$ illusion overall $=9.0 \%$ ). The illusion was larger for stimuli in the right hemispace $(M=11.7 \%)$ than for those in the left hemispace $(M=8.4 \%)$. However, an ANOVA on percentage of illusion scores showed a nonsignificant effect of group $(F<1)$. None of the other main effects or interaction effects reached significance (all $p \mathrm{~s}>.21$ ). An ANOVA on the size judgments also revealed a nonsignificant main effect of group $(F<1)$.

The illusion occurred in both hemispaces. Certainly, the attenuation of the illusion in Experiment 4 depended on placement at the body midline and free exploration. Midline placement allows individuals to feel their bodies with their elbows as they explore stimuli and to use the body as an effective spatial metric. In addition, the ability to maintain contact with the body may yield more stable and effective manual movements.

Conceivably, the illusion would be diminished if the stimuli were felt with one hand - for example, the right hand in the right hemispace. This would align the stimulus pattern with the shoulder, and there is some evidence that

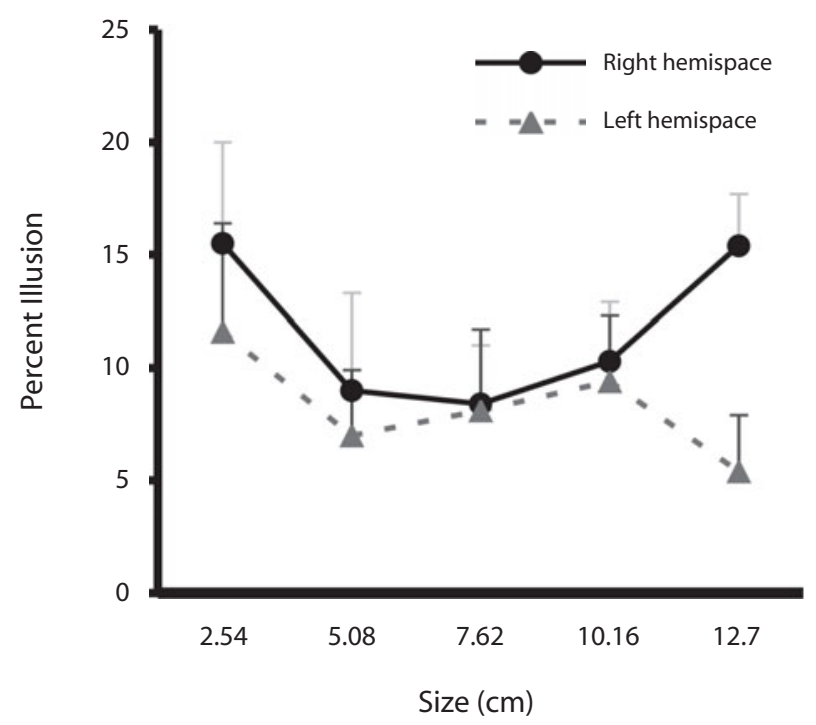

Figure 7. Percentage-of-illusion data for stimuli in the left and right hemispaces in Experiment 6. As in the earlier experiments, percent illusion was determined by subtracting the judged horizontal from the judged vertical $\times 2$, and dividing by the sum of the judged horizontal and vertical. These data reflect the strength of the illusion.

the haptic egocenter changes with the task (see Soechting \& Flanders, 1993; Soechting et al., 1990). Thus, alignment with a shoulder-centered frame of reference may reduce perceptual errors and diminish the illusion. Heller et al. (1997) used very different methods but did report a diminished horizontal-vertical illusion for inverted-T and L stimuli when they were felt and judged using the right hand in the right hemispace. The possible importance of stimulus alignment with the shoulder should be examined in future research. Millar and Al-Attar (2000) reported that the horizontal-vertical illusion was present when subjects felt stimuli with the right hand in the right hemispace. However, there was no indication of whether or not the stimuli were aligned with the shoulder in their report.

Another explanation of the results should be considered that derives from differences in the angles of the arms, especially the forearms, when they are at the midline, as compared with the left or right hemispaces. At the midline, the two arms will produce similar angles when feeling a single object at once. This changes when stimuli are in the left or right hemispace. In these circumstances, subjects experience differences in the angles generated by the two arms as they feel patterns with different movement patterns. This is a more complex situation than occurs when the configuration of the arms is symmetrical about the midline and could impair computation of extent. Furthermore, displacement of the stimulus from the midline and the location of the ruler at the midline further complicate distance estimates. On this interpretation of the results, one would expect diminished errors as a function of reductions in the distance of stimuli from the midline. Increases in the separation between stimuli and the midline should magnify errors and illusory misperception. Also, 
one might also expect that any displacement of the tangible rulers from the location of the stimuli would serve to magnify perceptual error and illusory distortion. This is properly the subject of future research.

\section{GENERAL DISCUSSION}

The results of the experiments confirm the presence of a robust horizontal-vertical illusion with convex curves in touch. It is important that the illusion was nearly eliminated when the stimuli were felt at the body midline with both hands (Experiment 4) but was present when the stimuli were freely explored with only one hand at the midline (Experiment 5) or with two index fingers at the midline (Experiment 4). The illusion was present throughout in Experiment 5, indicating that free exploration per se cannot explain the near elimination of the illusion in Experiment 4. Bimanual free exploration at the midline was critical, since the illusion was found when stimuli were explored by a single hand, even with free exploration. Moreover, the illusion was present when stimuli were felt with two hands in the left and right hemispace in Experiment 6.

Why, then, do people tend to overestimate the vertical dimensions of curves and underestimate the distance between their endpoints? One explanation involves the idea that subjects confuse distances as a consequence of increased tracing of curved extent. The results of Experiment 2 also showed the impact of radial scanning for touch, since the bases of the curves were judged as much longer when vertical (rotated) than when the bases were horizontal (the upright curves). These data are consistent with a movement-based heuristic explanation of the results (e.g., Lederman et al., 1985). Additional support for this view exists in the results of Experiment 4. Although bimanual exploration attenuated the illusion, it was not completely eliminated. In fact, the illusion was more than $5 \%$ for the largest, 12.7-cm stimuli. These large stimuli are difficult for many smaller subjects to grasp with their hands and may force tracing. Recall that the simple effect of group was nonsignificant in Experiment 4. Tracing was clearly a contributor to illusory misperception in these experiments.

The presence of a weak illusion for the largest stimuli in Experiment 4 and the absence of an illusion for the smaller stimuli are also consistent with the idea that scale is important for haptics. Haptics tends to yield more accurate perception with smaller stimuli and is not as capable as vision in dealing with very large stimuli. Thus, the horizontal-vertical illusion may diminish when confined to a hand space (e.g., Heller et al., 1997) but is much larger when arm motions occur as one feels a pattern. On this interpretation, one would expect a larger illusion with stimuli that exceed the maximum size that was studied in the present report. This effect of size would be expected, even with midline placement and bimanual exploration.

Tracing upright convex curves forces subjects to change direction at the curve's peak. This change in tracing direction may serve to bisect the curve for haptics and vision, much as in the inverted-T pattern. Perhaps this is a major contributor to the reduced size estimates for the horizontal distances between endpoints of the curves.
The data are consistent with some aspects of the suggestion by Künnapas (1955) for vision and by Heller and Joyner (1993; see also Cheng, 1968; Heller et al., 1997) for haptic stimuli. The visual field is oval and is wider than it is high. Thus, a vertical extent will take up a larger portion of the vertical field than a comparable extent will for a horizontal. This prompts overestimation of verticals. One can make the same argument for haptic manipulatory space. If one extends the two arms to the sides while seated, the available horizontal manipulatory space is larger than the vertical manipulatory space, owing to the separation of the two arms by the body. The availability of two arms for exploration contributes to this spatial inequality, especially for comparisons between large vertical and horizontal extents. This may yield similar overestimation of verticals in an oval haptic space (see Heller \& Joyner, 1993). This overestimation is likely when one hand explores stimuli at the midline or if the body midline is crossed when bimanually exploring forms away from the midline (see Soechting \& Flanders, 1993). Additional research is planned to evaluate the implications of this theoretical perspective. However, it does not seem completely convincing that reference to a larger horizontal arm space alone could explain the obtained robust illusion for very small patterns, like some of the smaller stimuli in the present experiments. Perhaps stimulus orientation interacts with exploration method, hemispace, and, possibly, hand effects to alter the illusion. This oval field explanation is not entirely consistent with the results of Experiment 2, given the minimal illusory distortion in judged height of the rotated bases with vision.

The results of Experiments 1, 2, and 4 suggest that some perceptual processes may be common across the modalities but that some may be modality specific. A visual illusion was found (see Figure 3) for curved stimuli that were flat on the table surface at the midline. The illusion seemed robust in vision, and this clearly warrants further investigation. The stimuli were rotated or upright in Experiment 2, and it is perhaps here that differences were revealed more clearly between vision and touch. The illusion was more potent in touch, and the results of rotation in vision differed from rotation of $\mathrm{T}$ shapes (see Landwehr, 2009). In vision, the vertical was no longer judged as much longer than the horizontal, given rotated curves with baselines.

The presence of apparently modality-specific processes appears problematic for ecological perspectives, but it is unlikely that these basic perceptual and philosophical controversies will be easy to resolve. The illusion was greatly diminished in Experiment 4 but was not eliminated for the largest stimuli. In Experiments 1 and 2, the illusion was present throughout but was absent for the smaller curves in haptics in Experiment 4. The lack of an illusion for the smaller stimuli does support the ecological view. Scale is an obvious limitation for touch. Moreover, although the visual and haptic illusions were reduced slightly in the frontal position in Experiment 1, the effect of position differed for the two senses as a function of size. Frontal placement reduced the illusion to a greater extent for the larger stimuli for vision, but not for touch. Size was judged differently by the senses of vision and touch. These ob- 
tained modality differences are not easy to reconcile with the ecological position, unless one adopts the theoretical view that the senses evolved to make relative, rather than absolute, judgments.

There are a few possible explanations for the effect of bimanual exploration at the midline that can be considered. One possibility is that the use of two hands at the midline allows individuals to use the body as a frame of reference or size metric for judging extent. The results are consistent with earlier reports of similar effects by Ballesteros and her colleagues in the study of symmetry (Ballesteros et al., 1998). The use of two hands practically eliminated the haptic Müller-Lyer illusion, even when the stimuli were felt with just two index fingers at the midline (Heller et al., 2005). Bimanual exploration at the midline may prompt more efficient and accurate exploration strategies and, hence, yield improved size estimates. For example, when using a single index finger for tracing Müller-Lyer stimuli, subjects rarely explored beyond the boundaries of horizontal lines with arrows pointing outward. This made them susceptible to the distracting, inhibitory effects of the arrows at the ends of the line. Bimanual exploration with two index fingers prompted line length estimates derived from feeling the outer tips of the arrow points. Heller et al. (2005) suggested that the use of two hands facilitated coding with respect to the body, since people can maintain contact with their bodies via the elbows as they feel stimuli at the body midline. This permits them to interpret felt size within the context of a body-scaled space. Presumably, people know how wide their bodies are and can use this metric knowledge to help them make size estimates. Note that the present results were not entirely consistent with those reported by Millar and Al-Attar (2000), since midline placement, alone, was not sufficient to eliminate the illusion in the present experiments.

Of course, there is ample evidence that many people do not have a proper understanding of how large or small their bodies may be. There is a large literature on distortion of the body image, even in people who are presumably normal and devoid of pathology of any sort (Shontz $\&$ McNish, 1972). Individual differences in knowledge of body size could be related to susceptibility to illusions. If people have distorted ideas about their body dimensions, they may also fail to accurately perceive the extent of an external object. Conversely, perhaps people with more accurate body size information will do better in judging the extent of nonbody objects. Also, when two hands can feel the stimuli at the midline, they provide additional spatial reference information for each other, in addition to any further spatial information provided by reference to the body itself. This synergistic effect of spatial reference information from the midline placement, combined with information derived from the two hands as a frame of reference, may also facilitate accurate size estimation.

Alternative explanations of the results were considered earlier, and these involve reference to the physical alignment of the arm or angles of the arms and shoulders when subjects feel the curved stimuli and when they feel the measuring device, the tangible ruler response measure. If subjects compute hand and arm orientation when explor- ing patterns and judging their sizes, this process should be easier when arm angle is identical for the two limbs. Thus, if one feels a stimulus to the left of the midline with both hands, arm angles will differ, and neither arm may be aligned with the shoulder. Alignment with the shoulder could aid perception (Soechting \& Flanders, 1993; Soechting et al., 1990). This factor of common arm angle may work in combination with altered exploration methods for the two hands at the midline. The midline placement may permit more efficient and different cooperative exploration strategies, as compared with exploration in the left or right hemispace.

What of the ideas presented by researchers in neuroscience, who have proposed that distinctions between perception and action can be revealed through the study of illusions such as the horizontal-vertical illusion? Servos, Carnahan, and Fedwick (2000) claimed that the perceptual system was fooled by the horizontal-vertical illusion but that reaching behavior yielded grip apertures that were veridical. Their results were described as consistent with the Goodale and Milner (1992) model that proposed separate neural pathways for perception and action. Again, this is a difficult issue to resolve, since people may not know how far apart the index finger and thumb are extended in a grasping posture. The index finger and thumb may simply act as pointers and indicators of spatial location, rather than a proper measure or judgment of extent (Smeets et al., 2002; see also Lederman et al., 1985). The subjects in Experiments 5 and 6 were able to use any method they wished to feel the patterns at the midline but were limited to one hand (Experiment 5) and were distant from the midline using both hands in Experiment 6. The subjects were free to use grasping, and many did so. Nonetheless, the illusion persisted. Future research should more carefully manipulate exploration methods to provide an answer to this question, since judgments of extent are different in important ways from judgments of position (Lederman et al., 1985). Note that the response measure in the present experiments mixed aspects of motor responding and perception. Nonetheless, the illusion was eliminated for the smaller stimuli in Experiment 4.

Curves are normal stimuli; however, curved tangible raised lines are artificial and may provide impoverished information when compared with curved 3-D surfaces. It remains to be seen whether or not identical illusory distortion can be demonstrated with curved lines and with curved solids. There are indications in the literature that this is likely, since similar illusory effects have been found for solid 3-D inverted-T and L shapes and raised lines (Heller et al., 2003; see also Vogels, Kappers, \& Koenderink, 1996).

Finally, the present results have significance for application. When visually impaired individuals are exposed to tangible maps, they need to be aware of the difficulties involved that may derive from distortion of the judgment of extent in curves. Maps are used to help visually impaired individuals make judgments about scale, landmarks, and directions. The presence of a horizontal-vertical illusion in curves is relevant, since maps may include these tangible patterns. Blind people should be encouraged to feel 
stimuli with both hands at the body midline, whenever possible.

\section{AUTHOR NOTE}

This research was supported by NSF Grant BCS-0317293 from the program in Perception, Action and Cognition. We are grateful to Smita Srivastava, Crystal Wanek, Karen Tate, Ashley Hasara, and Amy Matz for assistance with data collection and analyses. I am grateful to the anonymous reviewers for their very helpful, constructive comments. Correspondence concerning this article should be addressed to $\mathrm{M}$. A. Heller, Psychology Department, Eastern Illinois University, Physical Sciences Building, Charleston, IL 61920 (e-mail: maheller@eiu.edu).

\section{REFERENCES}

Amazeen, E. L., \& DaSilva, F. (2005). Psychophysical test for the independence of perception and action. Journal of Experimental Psychology: Human Perception \& Performance, 31, 170-182.

APPELle, S. (1991). Haptic perception of form: Activity and stimulus attributes. In M. A. Heller \& W. Schiff(Eds.), The psychology of touch (pp. 169-188). Hillsdale, NJ: Erlbaum.

Ballesteros, S., Manga, D., \& Reales, J. M. (1997). Haptic discrimination of bilateral symmetry in 2-dimensional and 3-dimensional unfamiliar displays. Perception \& Psychophysics, 59, 37-50.

Ballesteros, S., Millar, S., \& Reales, J. M. (1998). Symmetry in haptic and visual shape perception. Perception \& Psychophysics, 60, 389-404.

Cheng, M.-F. (1968). Tactile-kinesthetic perception of length. American Journal of Psychology, 81, 74-82.

Coren, S., \& Girgus, J. S. (1978). Seeing is deceiving: The psychology of visual illusions. Hillsdale, NJ: Erlbaum.

DAY, R. H., \& AvERY, G. C. (1970). Absence of the horizontal-vertical illusion in haptic space. Journal of Experimental Psychology, 83, 172-173.

DAY, R. H., \& Wong, T. S. (1971). Radial and tangential movement directions as determinants of the haptic illusion in an L figure. Journal of Experimental Psychology, 87, 19-22.

Faineteau, H., Gentaz, E., \& Viviani, P. (2003). The kinaesthetic perception of Euclidean distance: A study of the detour effect. Experimental Brain Research, 152, 166-172.

Faineteau, H., Gentaz, E., \& Viviani, P. (2005). Factors affecting the size of the detour effect in the kinaesthetic perception of Euclidean distance. Experimental Brain Research, 163, 503-514.

Gentaz, E., \& Hatwell, Y. (2004). Geometrical haptic illusions: The role of exploration in the Müller-Lyer, vertical-horizontal, and Delboeuf illusions. Psychonomic Bulletin \& Review, 11, 31-40.

GiBson, J. J. (1966). The senses considered as perceptual systems. Boston: Houghton Mifflin.

Gibson, J. J. (1979). The ecological approach to visual perception. Boston: Houghton Mifflin.

Goodale, M. A., \& Milner, A. D. (1992). Separate visual pathways for perception and action. Trends in Neurosciences, 15, 20-25.

Heelan, P. A. (1983). Space-perception and the philosophy of science. Berkeley: University of California Press.

Heller, M. A., Brackett, D. D., Salik, S. S., Scroggs, E., \& Green, S. (2003). Objects, raised-lines and the haptic horizontal-vertical illusion. Quarterly Journal of Experimental Psychology, 56A, 891-907.

Heller, M. A., Brackett, D. D., Wilson, K., Yoneyama, K., Boyer, A., \& StefFen, H. (2002). The haptic Müller-Lyer illusion in sighted and blind people. Perception, 31, 1263-1274.

Heller, M. A., Calcaterra, J. A., Burson, L. L., \& Green, S. L. (1997). The tactual horizontal-vertical illusion depends on radial motion of the entire arm. Perception \& Psychophysics, 59, 1297-1311.

Heller, M. A., Calcaterra, J. A., Green, S. L., \& Barnette, S. L. (1999). Perception of the horizontal and vertical in tangible displays: Minimal gender differences. Perception, 28, 387-394.

Heller, M. A., \& Joyner, T. D. (1993). Mechanisms in the haptic horizontal-vertical illusion: Evidence from sighted and blind subjects. Perception \& Psychophysics, 53, 422-428.

Heller, M. A., Joyner, T. D., \& DAN-Fodio, H. (1993). Laterality effects in the haptic horizontal-vertical illusion. Bulletin of the Psychonomic Society, 31, 440-442.
Heller, M. A., Kappers, A. M. L., McCarthy, M., Clark, A., RidDLe, T., FulKerson, E., ET AL. (2008). The effects of curvature on haptic judgments of extent in sighted and blind people. Perception, 37, 816-840

Heller, M. A., McCarthy, M., Schultz, J., Greene, J., Shanley, M., Clark, A., ET AL. (2005). The influence of exploration mode, orientation, and configuration on the haptic Müller-Lyer illusion. Perception, 34, 1475-1500.

Heller, M. A., Rogers, G. J., \& Perry, C. L. (1990). Tactile pattern recognition with the Optacon: Superior performance with active touch and the left hand. Neuropsychologia, 28, 1003-1006.

Hollins, M., \& Goble, A. K. (1988). Perception of the length of voluntary movements. Somatosensory Research, 5, 335-348.

JACKSON, R. E., \& CORMACK, L. K. (2008). Evolved navigation theory and the environmental vertical illusion. Evolution \& Human Behavior, 29, 299-304.

Jones, L. A., \& Lederman, S. J. (2006). Human hand function. New York: Oxford University Press.

Kennedy, J. M. (1993). Drawing and the blind: Pictures to touch. New Haven, CT: Yale University Press.

KÜNNAPAS, T. M. (1955). An analysis of the "vertical-horizontal illusion." Journal of Experimental Psychology, 49, 134-140.

LANDWEHR, K. (2009). Opposite haptic and visual induction effects observed with Titchener's $\perp$. Attention, Perception, \& Psychophysics, 71, 1197-1202.

Lederman, S. J., Klatzky, R. L., \& Barber, P. O. (1985). Spatial and movement-based heuristics for encoding pattern information through touch. Journal of Experimental Psychology: General, 114, 33-49.

McFarland, J., \& Soechting, J. F. (2007). Factors influencing the radial-tangential illusion In haptic perception. Experimental Brain Research, 178, 216-227.

Millar, S. (2006). Processing spatial information from touch and movement: Implications from and for neuroscience. In M. A. Heller \& S. Ballesteros (Eds.), Touch and blindness: Psychology and neuroscience (pp. 25-48). Mahwah, NJ: Erlbaum.

Millar, S., \& Al-AtTar, Z. (2000). Vertical and bisection bias in active touch. Perception, 29, 481-500.

Millar, S., \& Al-AtTar, Z. (2002). The Müller-Lyer illusion in touch and vision: Implications for multisensory processes. Perception \& Psychophysics, 64, 353-365.

Servos, P., Carnahan, H., \& Fedwick, J. (2000). The visuomotor system resists the horizontal-vertical illusion. Journal of Motor Behavior, 32, 400-404.

ShontZ, F. C., \& MCNish, R. D. (1972). The human body as stimulus object: Estimates of distances between body landmarks. Journal of Experimental Psychology, 95, 20-24.

Smeets, J. B. J., Brenner, E., de Grave, D. D. J., \& Cuijpers, R. H. (2002). Illusions in action: Consequences of inconsistent processing of spatial attributes. Experimental Brain Research, 147, 135-144.

Soechting, J. F., \& Flanders, M. (1993). Parallel, interdependent channels for location and orientation in sensorimotor transformations for reaching and grasping. Journal of Neurophysiology, 70, 1137-1150.

Soechting, J. F., \& Poizner, H. (2005). The use of motion cues in the haptic sense of circularity. Experimental Brain Research, 165, 413421 .

Soechting, J. F., Tillery, S. I. H., \& Flanders, M. (1990). Transformation from head- to shoulder-centered representation of target direction in arm movements. Journal of Cognitive Neuroscience, 2, $32-43$.

Summers, D. C., \& Lederman, S. J. (1990). Perceptual asymmetries in the somatosensory system: A dichhaptic experiment and critical review of the literature from 1929 to 1986. Cortex, 26, 201-226.

TEDFORD, W. H., JR., \& TUDOR, L. L. (1969). Tactual and visual illusions in the T-shaped figure. Journal of Experimental Psychology, 81, 199-201.

Vogels, I. M. L. C., Kappers, A. M. L., \& Koenderink, J. J. (1996). Haptic aftereffect of curved surfaces. Perception, 25, 109-119.

Wong, T. S. (1977). Dynamic properties of radial and tangential movements as determinants of the haptic horizontal-vertical illusion with an L-figure. Journal of Experimental Psychology: Human Perception \& Performance, 3, 151-164.

(Manuscript received December 7, 2008; revision accepted for publication April 12, 2010.) 